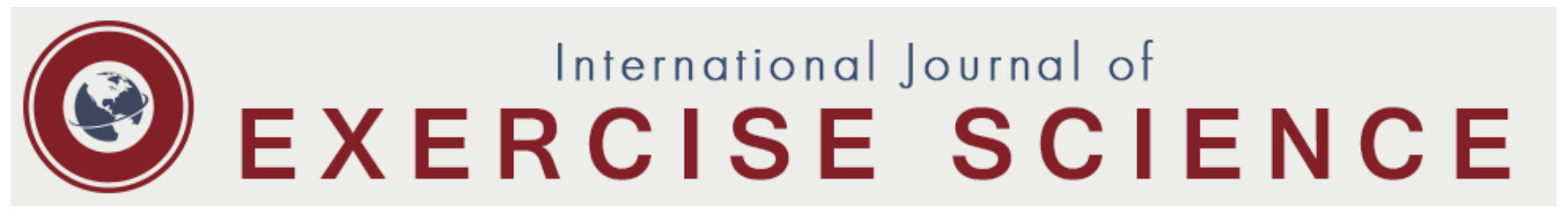

Original Research

\title{
Caffeine Alters RPE-Based Intensity Production
}

T. W. LANGFORD ${ }^{\dagger 1,2}$, E. K. O’NEAL ${ }^{\ddagger 1}$, E. M. SCUDAMORE ${ }^{\ddagger 1,3}$, M. C. STEVENSON ${ }^{\dagger 1}$, S. L. JOHNSON ${ }^{\ddagger 1,2}$, V. PRIBYSLAVSKA $\$ 1,3$, and J. M. GREEN $¥ 1$

1Department of Health, Physical Education, and Recreation, The University of North Alabama, Florence, AL, USA; ${ }^{2}$ Department of Health and Human Performance, Middle Tennessee State University, Murfreesboro, TN, USA; ${ }^{3}$ Health, Physical Education, and Sport Sciences, Arkansas State University, State University, AR, USA

*Denotes undergraduate student author, $†$ Denotes graduate student author, $\neq$ Denotes professional author

\section{ABSTRACT}

International Journal of Exercise Science 12(6): 412-424, 2019. This study examined effects of caffeine (CAF) on power output (PO) selection and associated physiological responses during cycling at moderate and high intensities prescribed by RPE (0-10 scale). Participants $(\mathrm{n}=9)$ (VO2peak: $55.4 \pm 6.32 \mathrm{~mL} \cdot \mathrm{kg}-1 \cdot \min -1)$ cycled for $20 \mathrm{~min}$ at RPE4 and $20 \mathrm{~min}$ at RPE7 separated by $10 \mathrm{~min}$ recovery following caffeine (CAF) $(6 \mathrm{mg} \cdot \mathrm{kg}-1)$ and placebo (PLA) ingestion. PO, HR, serum lactate [La], VO2, VE, and RER were recorded every 5 min. Session RPE (S-RPE) was recorded following 10 min recovery. Repeated-measures ANOVA's, 2 (trial) x 4 (time pt), showed significantly greater PO during RPE4 for CAF $(130 \pm 23 \mathrm{~W})$ vs PLA $(112 \pm 26 \mathrm{~W})$ and during RPE7 (CAF: $165 \pm 37$ vs PLA: $143 \pm$ $41 \mathrm{~W}$ ). Overall HR, VO2, and VE were significantly greater for CAF vs PLA during RPE7. RER for RPE4 and RPE7 were not significantly different (CAF vs PLA). Overall [La] was significantly greater for CAF during RPE4 (CAF: $2.32 \pm 0.94$ vs PLA: $1.73 \pm 1.09$ ) and RPE7 (CAF: $3.22 \pm 1.44$ vs PLA: $2.22 \pm 1.49$ ). Paired T-tests for S-RPE revealed no significant difference for RPE4 (CAF: $4.0 \pm 0.5$ vs PLA: $3.7 \pm 0.5$ ) or RPE7 (CAF: $7.1 \pm 0.3$ vs PLA: $6.9 \pm 0.6$ ) despite greater $\mathrm{PO}$ for $\mathrm{CAF}$. Although individual responses varied, the current study indicates caffeine ingestion results in elevated self-selected PO with significant systematic changes in associated physiological responses particularly at a higher intensity (RPE7).

KEY WORDS: Ergogenic aid, perceived exertion, RPE, performance

\section{INTRODUCTION}

Ergogenic effects of caffeine have been well established in aerobic $(26,28,30,31)$ and anaerobic $(4,14,20,34,36)$ exercise. A meta-analysis by Doherty and Smith (16) indicated caffeine ingestion prior to exercise improved performance by as much as $12 \%$ during endurance, graded, and short-term exercise tests. Although various dosages have been studied, oral consumption of anhydrous caffeine $1 \mathrm{hr}$ prior to exercise has been shown effective at $6 \mathrm{mg} \mathrm{kg}-1$ body mass (6, 13). 
Mechanisms by which caffeine may exhibit ergogenic effects continue to be explored. A competitive blockade of adenosine A1 and/or A2 receptors has been proposed $(24,51,55)$. Such a blockade in the central nervous system may lead to a decreased perception of pain during exercise $(7,14,18,45)$ resulting in greater effort, improved performance, and altered perceptual responses (RPE). Further, previous research has shown caffeine may alter RPE during exercise. RPE may be lower at a given workload following caffeine ingestion (6), or an effect can be demonstrated when RPE estimations are similar between trials when caffeine (vs placebo) results in completion of significantly more work $(11,37)$.

Traditionally, RPE may be estimated by a participant during activity reflecting subjective feelings of exertion or as a method for prescription and regulation of intensity (i.e. RPE production) (1). RPE-based prescriptions have been validated for laboratory settings using the estimation-production paradigm $(10,19,49,50)$. With this approach, individuals estimate RPE during an incremental exercise trial and in subsequent training, effort is adjusted to self-regulate intensity producing a target $\operatorname{RPE}(9,22,38)$. Although inconsistencies exist, RPE generally is effective for prescribing and regulating intensity $(23,32,33,42,52)$. Using the estimationproduction paradigm, measurement of $\mathrm{VO} 2$, lactate [La], heart rate (HR) or other objective physiological variables is not essential.

The influence of caffeine on RPE based intensity regulation (i.e. estimation-production paradigm) is not well understood, as effort sense during exercise remains a complex phenomenon (18). Cole et al (11) examined the effects of a $6 \mathrm{mg} \mathrm{kg}-1$ caffeine dose on the selfregulation of exercise intensity using three progressive RPE prescriptions. Participants performed a continuous $30 \mathrm{~min}$ cycling session subdivided into three $10 \mathrm{~min}$ bouts at prescribed RPE values $(9,12$, and 15). Compared to placebo, caffeine resulted in significantly greater total work for the combined $30 \mathrm{~min}$ exercise $(277.8 \pm 26.1 \mathrm{~kJ}$ vs $246.7 \pm 21.5 \mathrm{~kJ})$. Interestingly, workloads within levels of RPE prescription were not significantly different following caffeine ingestion. The potential influence of caffeine ingestion on self-selected workloads during RPE production trials is unclear. The current study examined effects of $6 \mathrm{mg} \mathrm{kg}-1$ caffeine on selfselected workload and associated physiological responses following RPE based intensity prescription.

\section{METHODS}

\section{Participants}

Male cyclists and runners were recruited $(n=9)$ as participants. Prior to data collection, participants completed a health screening questionnaire (PAR-Q) and a written, informed consent. Research was conducted in compliance with the guidelines and policies of the local Institutional Review Board for protection of human participants. During the initial lab session, descriptive data including age $(\mathrm{yr})$, height $(\mathrm{cm})$, and mass $(\mathrm{kg})$ were collected using a standard stadiometer (Detecto, Webb City, MO) and a calibrated Tanita EWB-800 scale (Tokyo, Japan). Body fat percentage was estimated via 3-skinfold-site method (47) (chest, abdomen, thigh) using Lange calipers (Cambridge, MA). A daily caffeine exposure questionnaire was administered to assess typical ingestion. Participants reported to the lab for a total of three sessions. Session 1 
included a maximal exertion test (VO2Peak) on a cycle ergometer (Monark 828E, Varberg, Sweden) and was separated from Session 2 by $\geq 48 \mathrm{hr}$. Sessions 2 and 3, caffeine (CAF) and placebo (PLA) sessions, were randomized, double-blind, and counterbalanced to control for ordering and separated by $\geq 24 \mathrm{hr}$. Time of testing for CAF and PLA was replicated for each trial within participants to limit effect of circadian rhythms on dependent measures. Participants were instructed to abstain from heavy exercise and caffeine use for $\geq 12 \mathrm{hr}$ prior to each testing session and instructed to record and replicate meals for the night before and day of testing for production trials.

\section{Protocol}

Prior to all testing, participants were fitted with an appropriately-sized air cushion mask (VacuMed, Ventura, CA) and HR monitor transmitter (T31 Transmitter, Polar Electro, Stamford, Kempele, Finland) before exercise. Metabolic data (VO2, VCO2, RER and VE) were assessed from expired air via an indirect open circuit spirometry system (Vacu-Med, Ventura, CA) equipped with Turbofit software (TurboFit, VacuMed, Ventura, CA). The system was calibrated prior to all metabolic testing using a gas of known concentration ( $16 \% \mathrm{O} 2,4 \% \mathrm{CO} 2)$. Ventilatory measurement was calibrated using a 3-L syringe (Hans Rudolph, Kansas City, MO). A 1-10 RPE scale (50) was displayed in view of participants at all times.

The Monark cycle ergometer was adjusted to individual preference for seat height and handlebar positioning. Participants were instructed to maintain 70 rev min- 1 throughout and began the exercise test at 35 Watts (W) for $3 \mathrm{~min}$ as a warm-up. Following the warm-up, resistance increased $70 \mathrm{~W}$ every 2 min until volitional exhaustion or failure of participant to maintain pedaling frequency for $10 \mathrm{~s}$ when provided verbal encouragement. Overall RPE (RPEO) was estimated by participants during the last $10 \mathrm{~s}$ of each minute. Metabolic data (20 s means) were reported during the last $10 \mathrm{~s}$ of each minute.

Caffeine and placebo (maltodextrin) capsules matched for appearance were administered in sealed containers prior to each trial. Ingestion of capsules at the appropriate time $(1 \mathrm{hr}$ prior to testing) was verified verbally upon arrival. During exercise trials, two capillary blood samples were taken from the fingertip and immediately analyzed for serum lactate concentrations [La] (1500 Sport Lactate Analyzer, Yellow Springs Instruments, Yellow Springs, OH). The mean of the two samples recorded at each time point was used for analysis. Prior to each trial, the analyzer was calibrated using $5 \mathrm{mmol}$ L-1 standard and checked for linearity with $15 \mathrm{mmol} \cdot \mathrm{L}-$ 1 standard as per manufacturer instructions.

During production trials, participants were instructed to maintain a pedaling frequency of 70 rev min-1 with cadence displayed digitally on the ergometer. Throughout exercise, participants were allowed to adjust the resistance on the ergometer; however, the readout display was concealed so that participants had no knowledge of the selected workload or PO. Participants began with a 3 min warm-up at $35 \mathrm{~W}$. Immediately following the warm-up, participants were allotted 3 min to adjust the resistance as needed to produce an RPE of 4 (RPE4) based on overall feelings of exertion. Following the 3 min production period, $\mathrm{PO}$ was recorded (time: $0 \mathrm{~min}$ ) and participants completed $20 \mathrm{~min}$ of cycling. Participants were only allowed to change the 
resistance every $5 \mathrm{~min}$, but were instructed to maintain the prescribed RPE. After cycling $20 \mathrm{~min}$, participants dismounted the ergometer and recovered passively for $10 \mathrm{~min}$. Following the 10 min recovery, participants estimated session RPE (S-RPE) and repeated the above protocol at a prescribed RPE of 7 (RPE7) based on overall feelings of exertion. At the completion of the RPE7 production trial, participants again recovered passively for $10 \mathrm{~min}$ following exercise termination and provided a S-RPE. Blood samples were collected and analyzed for [La] as described above at 5, 10, 15, and 20 min for RPE4 and RPE7. Corresponding metabolic data (60 s means) were also recorded at $5,10,15$, and $20 \mathrm{~min}$. HR was recorded continuously throughout each 20 min cycling trial (POLAR Team2 Pro, Polar Electro Oy, Kempele, Finland) and reported as $5 \mathrm{~min}$ means corresponding to [La] and metabolic data at 5, 10, 15 and $20 \mathrm{~min}$. PO was recorded at $0,5,10$, and $15 \mathrm{~min}$ with recording of physiological variables 5 min later per time point. This permitted a physiological steady state to be achieved which corresponded with the acute PO selection.

\section{Statistical Analysis}

All data were analyzed via SPSS software (Version 20). A series of repeated measures ANOVAs were used to assess differences with a principle focus on differences between treatments (CAF vs PLA). A 2 (trial) x 4 (time pt) repeated measures ANOVA was used for PO, HR, [La], VO2, RER, and VE with a principle focus on between trial differences at each time point. Tukey's LSD (1 tailed p-values) was used as a follow-up test between trials. Paired sample t-tests were used to analyze differences in S-RPE for RPE4 and RPE7. Results were considered significant at $\mathrm{p} \leq$ 0.05 . Based on the results of a similar study by Cole et al (11), a power analysis indicated that a sample size of 13 participants would be sufficient.

\section{RESULTS}

Mean and standard deviation for VO2, HR, [La], RER, and VE between CAF and PLA are displayed in Table 2 (RPE4) and Table 3 (RPE7). A significant main effect for trial for VO2 was found for RPE7 ( $p=0.01)$. Follow-up tests showed significantly greater values for CAF at 5,10 , 15 and $20 \mathrm{~min}$. The main effect for VO2 for RPE4 approached significance $(p=0.08)$. Follow-up tests showed significant differences at 5, 15 and $20 \mathrm{~min}$. Main effect for trial for HR was significant for RPE7 ( $p=0.03)$, but HR during RPE4 did not reach significance $(p=0.18)$. Followup tests showed HR during RPE7 was significantly greater for CAF at 10, 15 and 20 min, while during RPE4 significant differences were found at 15 and $20 \mathrm{~min}$. HR data for one participant during RPE4 trials were excluded due to equipment failure $(n=8)$. [La] was significantly greater for CAF vs PLA during both RPE4 (trial main effect, $p=0.03$ ) and RPE7 (trial main effect, $p=$ 0.03). [La] for CAF was significantly greater for RPE4 at 5 and $20 \mathrm{~min}$, while values for RPE7 were significant at 10, 15 and $20 \mathrm{~min}$ and approached significance at $5 \mathrm{~min}(\mathrm{p}=0.057)$. For RPE4, VE approached significance (trial main effect, $p=0.07$ ) with significance at 15 and $20 \mathrm{~min}$. Within RPE7 trials, VE was significantly greater for CAF vs PLA (trial main effect, $p=0.03$ ) with followup tests showing significance at $5,10,15$, and $20 \mathrm{~min}$. RER was not significantly different between treatments for either RPE4 $(p=0.68)$ or RPE7 $(p=0.64)$. There were no significant differences found for S-RPE for RPE4 (CAF $=4.0 \pm 0.5, \mathrm{PLA}=3.7 \pm 0.5, \mathrm{p}=0.20$ ) or RPE7 (CAF $=7.1 \pm 0.3$, PLA $=6.9 \pm 0.6, \mathrm{p}=0.35)$. 
Table 1. Descriptive characteristics for participants.

\begin{tabular}{lcc}
\hline Variable & Mean & SD \\
\hline Age $(\mathrm{yrs})$ & 28.2 & 6.5 \\
$\mathrm{Ht}(\mathrm{cm})$ & 176.6 & 5.6 \\
Mass $(\mathrm{kg})$ & 74.3 & 7.2 \\
Body fat $(\%)$ & 9.5 & 3.2 \\
$\mathrm{VO}_{2}$ peak $\left(\mathrm{mL} \cdot \mathrm{kg}^{-1} \mathrm{~min}^{-1}\right)$ & 55.4 & 6.3 \\
Mean daily caffeine consumption $(\mathrm{mg})$ & 257 & 174 \\
\hline
\end{tabular}

Follow-up tests showed significantly greater power selection at each time point for CAF for RPE4 (Figure 1) and RPE7 (Figure 2).

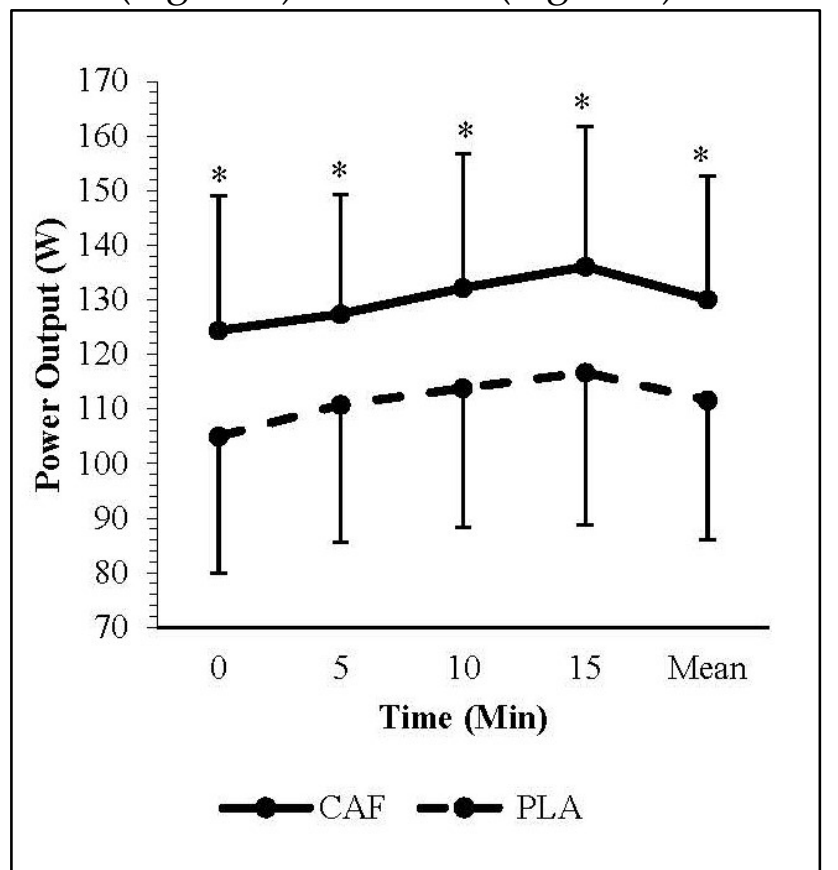

Figure 1. PO CAF vs PLA over time for $\mathrm{RPE}_{4}$

(Mean $\pm \mathrm{SD}) .{ }^{*} \mathrm{p} \leq 0.05$.

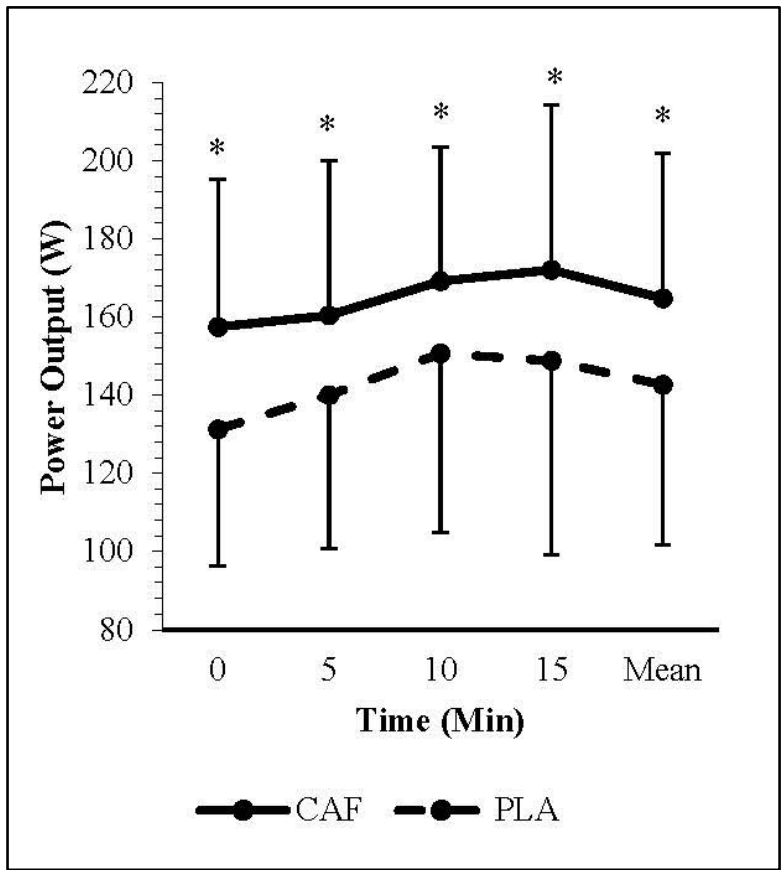

Figure 2. PO CAF vs PLA over time for RPE7 (Mean $\pm \mathrm{SD}) .{ }^{*} \mathrm{p} \leq 0.05$.

Table 2. Physiological responses for CAF vs. PLA for $\mathrm{RPE}_{4}$ over time (Mean $\pm \mathrm{SD}$ ).

\begin{tabular}{|c|c|c|c|c|c|}
\hline & Trial & $5 \mathrm{~min}$ & $10 \mathrm{~min}$ & $15 \mathrm{~min}$ & $20 \mathrm{~min}$ \\
\hline \multirow[t]{2}{*}{$\mathrm{VO}_{2}\left(\mathrm{~mL} \cdot \mathrm{kg}^{-1} \mathrm{~min}^{-1}\right)$} & CAF & $30.0 \pm 5.0^{*}$ & $31.0 \pm 4.7 \dagger$ & $31.6 \pm 4.5^{*}$ & $32.4 \pm 5.2^{*}$ \\
\hline & PLA & $25.1 \pm 3.7$ & $26.7 \pm 5.8$ & $27.6 \pm 5.5$ & $27.8 \pm 6.3$ \\
\hline \multirow{2}{*}{$\mathrm{HR}\left(\mathrm{b} \min ^{-1}\right)$} & CAF & $132 \pm 16$ & $137 \pm 18$ & $141 \pm 18 \dagger$ & $144 \pm 20^{*}$ \\
\hline & PLA & $127 \pm 13$ & $128 \pm 14$ & $131 \pm 14$ & $132 \pm 15$ \\
\hline \multirow{2}{*}{ [La] $\left(\mathrm{mmol} \cdot \mathrm{L}^{-1}\right)$} & CAF & $2.44 \pm 0.54^{*}$ & $2.16 \pm 0.97$ & $2.30 \pm 1.23 \dagger$ & $2.39 \pm 1.31^{*}$ \\
\hline & PLA & $1.78 \pm 0.70$ & $1.70 \pm 1.24$ & $1.67 \pm 1.25$ & $1.75 \pm 1.30$ \\
\hline \multirow{2}{*}{$V_{\mathrm{E}}\left(\mathrm{L} \cdot \min ^{-1}\right)$} & CAF & $43.8 \pm 6.0$ & $45.9 \pm 6.8 \dagger$ & $47.36 \pm 5.7^{*}$ & $48.78 \pm 7.8^{*}$ \\
\hline & PLA & $39.2 \pm 7.4$ & $39.7 \pm 7.5$ & $41.03 \pm 7.9$ & $42.61 \pm 8.8$ \\
\hline \multirow{2}{*}{ RER } & CAF & $0.88 \pm 0.06$ & $0.87 \pm 0.05$ & $0.89 \pm 0.05$ & $0.88 \pm 0.05$ \\
\hline & PLA & $0.91 \pm 0.09$ & $0.89 \pm 0.05$ & $0.89 \pm 0.05$ & $0.91 \pm 0.07$ \\
\hline
\end{tabular}

${ }^{*} \mathrm{p} \leq 0.05$ CAF vs PLA. $\dagger \mathrm{p}=0.09$ CAF vs PLA. 
Table 3. Physiological responses for CAF vs. PLA for $\mathrm{RPE}_{7}$ over time (Mean $\pm \mathrm{SD}$ ).

\begin{tabular}{|c|c|c|c|c|c|}
\hline & Trial & $5 \mathrm{~min}$ & $10 \min$ & $15 \mathrm{~min}$ & $20 \mathrm{~min}$ \\
\hline \multirow{2}{*}{$\mathrm{VO}_{2}\left(\mathrm{~mL} \cdot \mathrm{kg}^{-1} \mathrm{~min}^{-1}\right)$} & CAF & $35.3 \pm 5.2^{*}$ & $37.5 \pm 5.3^{*}$ & $39.1 \pm 5.6^{*}$ & $39.6 \pm 4.6^{*}$ \\
\hline & PLA & $29.8 \pm 5.5$ & $32.0 \pm 4.9$ & $33.9 \pm 6.6$ & $33.4 \pm 7.7$ \\
\hline \multirow{2}{*}{$\mathrm{HR}\left(\mathrm{b} \cdot \min ^{-1}\right)$} & CAF & $145 \pm 18 \dagger$ & $154 \pm 17^{*}$ & $160 \pm 17^{*}$ & $164 \pm 17^{*}$ \\
\hline & PLA & $136 \pm 19$ & $140 \pm 20$ & $146 \pm 20$ & $150 \pm 21$ \\
\hline \multirow{2}{*}{ [La] $\left(\mathrm{mmol} \cdot \mathrm{L}^{-1}\right)$} & CAF & $2.55 \pm 1.3^{* *}$ & $2.90 \pm 1.50^{*}$ & $3.46 \pm 1.64^{*}$ & $3.96 \pm 1.66^{*}$ \\
\hline & PLA & $1.99 \pm 1.4$ & $2.02 \pm 1.60$ & $2.41 \pm 1.71$ & $2.45 \pm 1.44$ \\
\hline \multirow{2}{*}{$\mathrm{V}_{\mathrm{E}}\left(\mathrm{L} \cdot \mathrm{min}^{-1}\right)$} & CAF & $56.5 \pm 12.3^{*}$ & $59.7 \pm 9.4^{*}$ & $65.4 \pm 9.7^{*}$ & $68.6 \pm 10.4^{*}$ \\
\hline & PLA & $45.1 \pm 8.1$ & $48.8 \pm 8.6$ & $54.0 \pm 12.0$ & $54.6 \pm 12.6$ \\
\hline \multirow{2}{*}{ RER } & CAF & $0.91 \pm 0.07$ & $0.93 \pm 0.04$ & $0.95 \pm 0.05$ & $0.95 \pm 0.05$ \\
\hline & PLA & $0.91 \pm 0.06$ & $0.91 \pm 0.05$ & $0.94 \pm 0.06$ & $0.93 \pm 0.07$ \\
\hline
\end{tabular}

${ }^{*} p \leq 0.05$ CAF vs PLA. ** $p=0.57$ CAF vs PLA. $\dagger p=0.09$ CAF vs PLA.

\section{DISCUSSION}

Previous research indicates that caffeine attenuates estimated RPE, potentially associated with improved exercise performance $(2,16,46)$. RPE may be mitigated when exercising at a given workload compared to placebo $(7,17,41)$ or RPE may be similar (vs placebo) when greater amounts of work are performed $(15,37,43)$. Both paradigms reflect an effect of caffeine on RPE. In regards to training, prescribing exercise utilizing RPE (RPE production) is well accepted (1, 39). Advantages include convenience due to a decreased need for assessing objective measures such as HR, [La] or VO2. It is unclear if the mitigating effects of a moderate dose of caffeine persist when exercise intensity is prescribed and regulated using RPE. Therefore, this study examined effects of $6 \mathrm{mg} \mathrm{kg}-1$ caffeine (vs placebo) on workload selection and associated physiological responses following exercise intensity prescriptions of RPE 4 and 7.

The main finding of the current study was that the ingestion of caffeine resulted in the selfselection of significantly higher workloads across time during lower (RPE4) (Figure 1) and higher (RPE7) (Figure 2) intensity production trials. A similar study by Cole et al (11) reported caffeine did not result in a significant increase in mean work output during RPE production (RPE: 9, 12, and 15), but resulted in significantly higher accumulated work. It was suggested that the increase in work output following caffeine ingestion was not dependent upon the RPE level. In contrast, current results suggest caffeine significantly increased self-selected PO during both RPE4 (CAF: $130.0 \pm 22.6 \mathrm{~W}$, PLA: $111.6 \pm 25.5 \mathrm{~W}$ ) and RPE7 (CAF: $164.8 \pm 37.0 \mathrm{~W}$, PLA: $142.7 \pm$ $40.9 \mathrm{~W}$ ). Although both studies showed a significant increase in total work, discrepancy between studies may be attributable to the protocol used for RPE prescription. Cole et al. (11) utilized a 30 min cycling exercise protocol consisting of three progressive levels of RPE (10 min each). The current study used an introductory 3 min production period, during this period participants were able to closely ensure their individual workload pertaining to each RPE level (4 and 7) prior to a 20 min cycling trial. This may be a source of the discrepancy since individual PO was selected to the target RPE before data collection commenced. Therefore, for RPE production during cycling exercise preceded by caffeine ingestion, the production period duration and protocol may impact acute outcomes. 
Caffeine is known to limit the binding of endogenous adenosine to adenosine receptors reducing adenosine's natural suppression of arousal and inhibitory modulation of neuronal excitability $(24,51)$. Therefore, it is plausible that caffeine acted as an analgesic reducing afferent feedback of exercise-associated pain and discomfort during production trials in the current study, resulting in selection of greater workloads. That is, responses observed for PO appear to have been due to the requirement of a greater stimulus (i.e. higher workload) to yield the prescribed RPE.

In the current study, participants were blinded to acute PO during production trials and intensity was regulated by gauging overall subjective feelings throughout twenty minute cycling bouts. During performance based paradigms (i.e. time-trials), caffeine has increased selfselected intensities and improved exercise performance as participants were aware of the performance based nature of the test $(8,12,15,40)$. Contrary to the nature of those studies, the current paradigm did not guide participants with any performance-based incentives; instead participants were instructed to maintain the prescribed intensity (RPE 4 and 7) as in a daily bout of exercise training. Consistent selection of higher workloads may increase total workload performed during daily exercise, which could result in greater total caloric expenditure during exercise sessions and enhanced intensity-dependent training adaptations.

Duncan et al. (21) reported that caffeine ingestion elevated subjective measures of participant readiness to invest both mental and physical effort after performing fatiguing tests and prior to exercise performance tasks. If this occurred in the current study, a caffeine-induced elevation in the motivational state of participants may have altered subjective feelings of intensity (18). In fact, 6 out of 9 participants selected a higher initial PO following caffeine compared to placebo ingestion. This may be an important consideration when caffeine consumption precedes RPEregulated exercise training. However, current participants were not asked to identify CAF and PLA treatments and no attempt was made to directly assess motivational status. Regardless, ingestion of caffeine resulted in ergogenic benefits plausibly attributable to an alteration in RPEworkload congruence. Future research should more directly examine potential effects of caffeine on motivational status and performance during perceptually-regulated training.

Table 2 shows consistently higher VO2, HR, and VE, values for CAF compared to PLA during RPE4. Failure to achieve overall statistical significance for these variables may have been due to the relatively low intensity associated with RPE4. At a lower intensity, there may be a relatively low volume of pain, discomfort and change in other factors believed to mediate RPE. That is, if altered pain is the mechanism of action for caffeine, it is reasonable that low intensity exercise (vs higher intensity) presents a weaker paradigm in which to observe a convincing effect. A liberal approach to conducting follow-up tests was taken in the interest of identifying an effect, if it exists. More specifically, follow-up tests were conducted at specific time points for physiological variables when main effects approached significance because $\mathrm{PO}$ values were significant. More research is warranted as follow-up analyses revealed significantly greater VO2 values for CAF compared to PLA at 5, 15 and $20 \mathrm{~min}$ (Table 2). 
Robertson et al (48) contended that HR may not be an important mediator of exertional perception, while ventilatory drive stands to be a potent and consciously monitored physiological mediator for respiratory-metabolic perceptual signals during dynamic exercise. Exertional perceptions during exercise may also be influenced by peripheral afferent input (48). In contention, Marcora et al (44) expressed that afferent feedback from locomotor and respiratory muscles lacked a significant contribution to RPE. Current results show significantly greater HR and VE (RPE7) for CAF vs PLA, while greater workloads were selected to maintain the prescribed RPE (Table 3). Therefore, HR and VE as physiological mediators of exertional perception may have been suppressed due to the effects of CAF, while peripheral afferent feedback may have been masked as CAF prompted the requirement of greater workloads during exercise to produce the prescribed RPE (Table 3). It is understood that RPE is not mediated by any single physiological or psychological variable and in the current study contributions from independent mediators could not be discerned. However, it would seem that during low intensity exercise following caffeine ingestion, peripheral afferent input from exercising muscles may serve as a more important mediator of RPE due to the lack of response from HR, VE, and VO2. Further, mediators of RPE appear to be less distinguished during a moderate (vs higher) exercise intensity. More work is warranted to extend the knowledge in this regard.

It was hypothesized that any significant increases in self-selected PO would subsequently elicit a response in [La]. Overall [La] during RPE4 was significantly greater for CAF vs PLA (Table 2), which concurs with other studies investigating the ergogenic effects of caffeine $(5,6,40,54)$. However, selection of greater workloads for CAF at RPE4 was only associated with significantly higher [La] for CAF vs PLA at 5 and 20 min (Table 2). [La] measurements for RPE7 (Table 3) were significantly greater for CAF compared to PLA with follow-up analyses showing significance at 10, 15 and $20 \mathrm{~min}$, while approaching significance at $5 \mathrm{~min}(\mathrm{p}=0.057)$. In comparison to the lower intensity (RPE4), CAF manifested a greater effect at the higher intensity (RPE7) as [La] remained significantly elevated following the selection of higher power outputs. Future research should attempt to examine whether caffeine acted peripherally or centrally to affect metabolic changes or muscle recruitment.

While caffeine's influence on free fatty acids (FFA) mobilization have been debated, the observed RER values were not expressive of a turbulent shift to fat utilization during either RPE4 (Table 2) or RPE7 (Table 3) trials. Previous research has shown caffeine to function as an ergogenic aid by extending time to fatigue with benefits attributed to enhanced FFA concentration $(3,37,53)$. However, enhanced performance has been observed in absence of increased FFA $(27,29,35)$ indicating benefits of caffeine do not solely rest on altered substrate availability. In the current paradigm, RER values were either similar or lower for CAF versus PLA (Tables 2 and 3) even though PO was greater for CAF. This suggests a possible increased reliance on fat utilization, however, direct measures of blood FFA were not assessed.

S-RPE involves subjective estimation of effort pertaining to the global difficulty of an entire exercise session. S-RPE estimation following exercise serves as a model for monitoring training responses and preventing the possible adverse effects of overtraining (25). Caffeine ingestion 
has been shown to attenuate S-RPE even when total work volume is equated between caffeine and placebo trials (41). Current results revealed no significant difference for S-RPE (CAF vs PLA) for RPE4 (Table 2) or RPE7 (Table 3). Different from the constant-load exercise protocol with equated total work volume in Killen et al (41), during the current study RPE remained constant between CAF and PLA conditions yet selection of PO was greater for CAF (vs PLA). Therefore, the similar S-RPE (CAF vs PLA) observed with concurrent higher PO indicates caffeine also altered S-RPE. A systematic increase would have been expected for S-RPE yet this was not observed. Consequently, current results confirm those of Killen et al. (41) that caffeine alters SRPE responses.

A practical difference in $\mathrm{PO}$ was assumed to be a mean difference $\geq 20 \mathrm{~W}$. Using this criterion, 3 participants were identified as non-responders and 2 as negative responders for RPE4. For RPE7, 3 participants were identified as non-responders. This observation supports the notion that individual responses vary. Following the removal of non-responders, the relative intensity based on \%VO2Peak for each individual was calculated. Figure 3 and Figure 4 display calculated data (Mean $\pm \mathrm{SD}$ ) of responders for achieved percent relative VO2Peak (\%VO2Peak) compared between CAF and PLA for RPE4 and RPE7, respectively. At RPE4, responders exercised at a mean \%VO2Peak at least 15\% greater during CAF than PLA. At RPE7, mean \%VO2Peak for responders was at least $13 \%$ greater (CAF vs PLA). Assessment of individual results is important to ascertain the true impact of caffeine. From a statistical standpoint, those who fail to respond for whatever reason will alter the overall aggregate results and thus attenuate the magnitude of the impact for those who do respond. Further work is needed to determine the precise reasons for variation in responses among individuals.
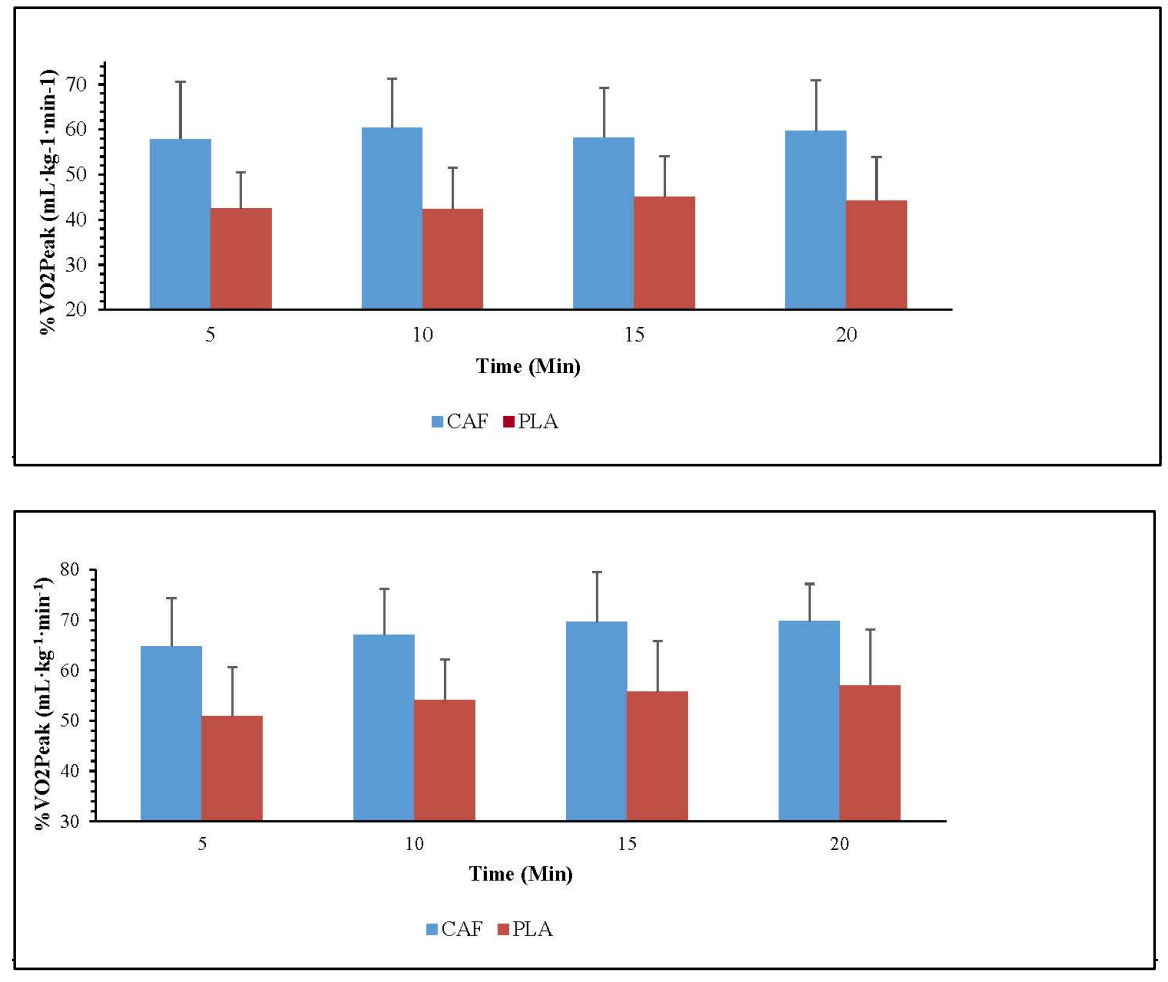

Figure. 3 Mean relative $\% \mathrm{VO}_{2 \text { Peak }}$ CAF vs PLA over time for $\mathrm{RPE}_{4}$ for responders only $(n=4)$.

Figure 4. Mean relative $\% \mathrm{VO}_{2 \text { Peak }}$ CAF vs PLA over time for $\mathrm{RPE}_{7}$ for responders only $(n=6)$. 
RPE production is a validated and useful method for prescribing exercise that does not require monitoring of physiological variables such as HR. The results of this study show that a moderate dose of caffeine ingested $1 \mathrm{hr}$ prior to exercise may alter an individual's perception of effort sequentially increasing total work during cycling exercise. Based on the current RPE-production paradigm, individuals ingesting caffeine prior to exercise may benefit from increased total work performed without a noticeable increase in perception of effort. Future research should more directly examine potential effects of caffeine on motivational status and performance during perceptually-regulated training. Although individual variation exists, it should be noted that RPE-based exercise prescription may be affected by caffeine consumption.

Current results indicate caffeine ingestion $(6 \mathrm{mg} \mathrm{kg}-1)$ resulted in an increase in PO selection during RPE-based exercise prescription. Associated physiological variables also changed concomitant with elevated PO. While direct mechanisms associated with the observed differences are not clear, results have implications with regard to RPE-based exercise prescriptions. Although the RPE production paradigm is useful, efficacy following the ingestion of an ergogenic aid such as caffeine is not well understood. Understanding the link between perceptual responses and caffeine is important due to the increasing utilization of caffeine as an ergogenic aid prior to or during exercise. Notable variations in responses among participants highlight the importance of assessing individual responses to specific ergogenic aids.

\section{REFERENCES}

1. American College of Sports Medicine. Guidelines for Exercise Testing and Prescription, 9th edition. Baltimore, MD, Williams and Wilkins. 2014.

2. Astorino TA, Cottrell T, Lozano AT, Aburto-Pratt K, Duhan J. Increases in cycling performance in response to caffeine ingestion are repeatable. Nutr Res 32: 78-84, 2012.

3. Bell DG, Jacobs I, Zamecnik J. Effects of caffeine, ephedrine and their combination on time to exhaustion during high-intensity exercise. Eur J Appl Physiol 77: 427-433, 1998.

4. Bell DG, Jacobs I, Ellerington K. Effect of caffeine and ephedrine ingestion on anaerobic exercise performance. Med Sci Sports Exerc 33(8): 1399-1403, 2001.

5. Bell DG, McLellan TM. Exercise endurance 1, 3, and $6 \mathrm{~h}$ after caffeine ingestion in caffeine users and nonusers. J Appl Physiol 93: 1227-1234, 2002.

6. Bell DG, McLellan TM. Effect of repeated caffeine ingestion on repeated exhaustive exercise endurance. Med Sci Sports Exerc 35(8): 1348-1354, 2003.

7. Birnbaum LJ, Herbst JD. Physiologic effects of caffeine on cross-country runners. J Strength Cond 18(3): 463-465, 2004.

8. Bridge CA, Jones MA. The effect of caffeine ingestion on $8 \mathrm{~km}$ run performance in a field setting. J Sports Sci 24(4): 433-439, 2006.

9. Buckley JP, Eston RG, Sim J. Ratings of perceived exertion in braille: validity and reliability in production mode. Br J Sports Med 34: 297-302, 2000. 
10. Ceci R, Hassmen P. Self-monitored exercise at three different RPE intensities in treadmill vs. field running. Med Sci Sports Exerc 23(6): 732-738, 1991.

11. Cole KJ, Costill DL, Starlin RD, Godpaster BH, Trappe SW, Fink WJ. Effect of caffeine ingestion on perception of effort and subsequent work. Int J Sports Nutr 6: 14-23, 1996.

12. Conway KJ, Orr R, Stannard SR. Effect of A divided caffeine dose on endurance cycling performance, postexercise urinary caffeine concentration, and plasma paraxanthine. J Appl Physiol 94: 1557-1562, 2003.

13. Cox GR, Desbrow B, Montgomery PG, Anderson ME, Bruce CR, Macrides TA, Martin DT, Moquin A, Roberts A, Hawley JA, Burke LM. Effect of different protocols of caffeine intake on metabolism and endurance performance. J Appl Physiol 93: 990-992, 2002.

14. Davis JK, Green JM. Caffeine and anaerobic performance: ergogenic value and mechanisms of action. Sports Med 39: 813-832, 2009.

15. Desbrow B, Biddulph C, Devlin B, Grant GD, Anoopkumar-Dukie S, Leveritt MD. The effects of different doses of caffeine on endurance cycling time trial performance. J Sports Sci 30(2): 115-120, 2012.

16. Doherty M, Smith PM. Effects of caffeine ingestion on exercise testing: a meta-analysis. Int J Sports Nutr Exerc Metab 14: 626-646, 2004.

17. Doherty M, Smith PM, Hughes MG, Davison RC. Caffeine lowers perceptual response and increases power output during high-intensity cycling. J Sports Sci 22: 637-643, 2004.

18. Doherty M, Smith PM. Effects of caffeine on rating of perceived exertion during and after exercise: a metaanalysis. Scand J Med Sci Sports 15: 69-78, 2005.

19. Dunbar CC, Robertson RJ, Baun R, Blandin MF, Metz K, Burdett R, Goss FL. The validity of regulating exercise intensity by ratings of perceived exertion. Med Sci Sports Exerc 24(1): 94-99, 1992.

20. Duncan MJ and Oxford SW. The effect of caffeine ingestion on mood state and bench press performance to failure. J Strength Cond Res 25(1): 178-185, 2011.

21. Duncan MJ, Smith M, Cook K, James RS. The acute effect of a caffeine-containing drink on mood state, readiness to invest effort, and resistance exercise to failure. J Strength Cond Res 26(10): 2858-2865, 2012.

22. Eston RG, Williams JG. Reliability of ratings of perceived effort regulation of exercise intensity. Br J Sports Med 22(4): 153-155, 1988.

23. Faulkner J, Eston RG. Perceived exertion research in the 21st century: developments, reflection, and questions for the future. J Exerc Sci Fit 6(1): 1-14, 2008.

24. Fredholm BB, Battig K, Holmen J, Nehlig A, Zvartau EE. Actions of caffeine in the brain with special reference to factors that contribute to its widespread use. Pharmacol Rev 51: 83-133, 1999.

25. Foster C, Florhaug JA, Franklin J, Gottschall L, Hrovatin LA, Parker S, Doleshal P, Dodge C. A new approach to monitoring exercise training. J Strength Cond Res 15: 109-115, 2001.

26. Goldstein ER, Ziegenfuss T, Kalman D, Kreider R, Campbell B, Willborn C, Taylor L, Willoughby D, Stout J, Graves BS, Wildman R, Ivy JL, Spano M, Smith AE, Antonio J. International society of sports nutrition position stand: caffeine and performance. J Int Soc Sports Nutr 7: 5, 2010. 
27. Graham TE, Spriet LL. Performance and metabolic responses to a high caffeine dose during prolonged exercise. J Appl Physiol 71(6): 2292-2298, 1991.

28. Graham TE, Spriet LL. Caffeine and exercise performance. Sports Science Exchange. Ontario, CA: Gatorade Sports Science Institute. 9(1), 1996.

29. Graham TE, Helge JW, MacLean DA, Kiens B, Richter EA. Caffeine ingestion does not alter carbohydrate or fat metabolism in human skeletal muscle during exercise. J Physiol 529(3): 837-847, 2000.

30. Graham TE. Caffeine, coffee and ephedrine: impact on exercise performance and metabolism. Can J Appl Physiol 26: S103-S119, 2001.

31. Graham TE. Caffeine and exercise: metabolism, endurance and performance. Sports Med 31(11): 785-807, 2001.

32. Green JM, Michael T, Solomon AH. The validity of ratings of perceived exertion for cross-modal regulation of swimming intensity. J Sports Med Phys Fitness 39(3): 207-215, 1999.

33. Green JM, Crews TR, Pritchett RC, Mathfield C, Hall L. Heart rate and ratings of perceived exertion during treadmill and elliptical exercise training. Percept Mot Skills 98(1): 340-348, 2004.

34. Green JM, Wickwire PJ, McLester JR, Gendle S, Hudson G, Pritchett RC, Laurent CM. Effects of caffeine on repetitions to failure and ratings of perceived exertion during resistance training. Int J Sports Physiol Perf 2: 250$259,2007$.

35. Greer F, Friars D, Graham TE. Comparison of caffeine and theophylline ingestion: exercise metabolism and endurance. J Appl Physiol 89: 1837-1844, 2000.

36. Hudson GM, Green JM, Bishop PA, Richardson MT. Effects of caffeine and aspirin on light resistance training performance, perceived exertion, and pain perception. J Strength Cond Res 22(6): 1950-1957, 2008.

37. Ivy JL, Costill DL, Fink WJ. Influence of caffeine and carbohydrate feedings on endurance performance. Med Sci Sports 11(1): 6-11, 1979.

38. Kang J, Hoffman JR, Walker H, Chaloupka EC, Utter AC. Regulating intensity using perceived exertion during extended exercise periods. Eur J Appl Physiol 89: 475-482, 2003.

39. Kang J, Chaloupka EC, Biren GB, Mastrangelo MA, Hoffman JR. Regulating intensity using perceived exertion: effect of exercise duration. Eur J Appl Physiol 105: 445-551, 2009.

40. Kovacs EMR, Stegen JHCH, Brouns F. Effect of caffeinated drinks on substrate metabolism, caffeine excretion, and performance. J Appl Physiol 85: 709-715, 1998.

41. Killen LG, Green JM, O'Neal EK, McIntosh JR, Hornsby J, Coates TE. Effects of caffeine on session ratings of perceived exertion. Eur J Appl Physiol 113: 721-727, 2013.

42. Lambrick DM, Faulkner JA, Rowlands AV, Eston RG. Prediction of maximal oxygen uptake from submaximal ratings of perceived exertion and heart rate during a continuous exercise test: the efficacy of RPE 13. Eur J Appl Physiol 107: 1-9, 2009.

43. Laurence G, Wallman K, Guelfi K. Effects of caffeine on time trial performance in sedentary men. J Sports Sci 30(12): 1235-1240, 2012. 
44. Marcora S. Perception of effort during exercise is independent of afferent feedback from skeletal muscles, heart, and lungs. J Appl Physiol 106: 2060-62, 2009.

45. McNaughton LR. The influence of caffeine ingestion on incremental treadmill running. Br J Sports Med 20(3): 109-112, 1986.

46. Norager $C B$, Jensen MB, Madsen MR, Laurberg S. Caffeine improves endurance in 75-yr-old citizens: a randomized, double-blind, placebo-controlled, crossover study. J Appl Physiol 99: 2302-2306, 2005.

47. Pollock ML, Schmidt DH, Jackson AS. Measurement of cardiorespiratory fitness and body composition in the clinical setting. Compr Ther 6(9): 12-27, 1980.

48. Robertson RJ, Nixon PA, Casperson CJ, Metz KF, Abbott R, Goss FL. Abatement of exertional perceptions following dynamic exercise: physiological mediators. Med Sci Sports Exerc 24(3): 346-353, 1992.

49. Robertson RJ, Noble BJ. Perception of physical exertion: methods, mediators, and applications. Exerc Sport Sci Rev 25(1): 407-452, 1997.

50. Robertson RJ, Goss FL, Dube J, Rutkowski J, Dupain M, Brennan C, Andreacci. Validation of the adult OMNI scale of perceived exertion for cycle ergometer exercise. Med Sci Sports Exerc 36(1): 102-108, 2004.

51. Sawynok J, Yaksh TL. Caffeine as an analgesic adjuvant: A review of pharmacology and mechanisms of action. Pharmacol Rev 45(1): 43-85, 1993.

52. Schafer MA, Goss FL, Robertson RJ, Nagle-Stilley EF, Kim K. Intensity selection and regulation using the OMNI scale of perceived exertion during intermittent exercise. Appl Physiol Nutr Metab 38(9): 960-966, 2013.

53. Spriet LL, MacLean DA, Dyck DJ, Hultman E, Cederblad G, Graham TE. Caffeine ingestion and muscle metabolism during prolonged exercise in humans. Am J Physiol 262(6): E891-898, 1992.

54. Talanian JL, Spriet LL. Low doses of caffeine late in exercise improve cycling time trial performance. FASEB J 21: 107(abstract), 2007.

55. Zhang W. A benefit-risk assessment of caffeine as an analgesic adjuvant. Drug Safety 24(15): 1127-1142, 2001.
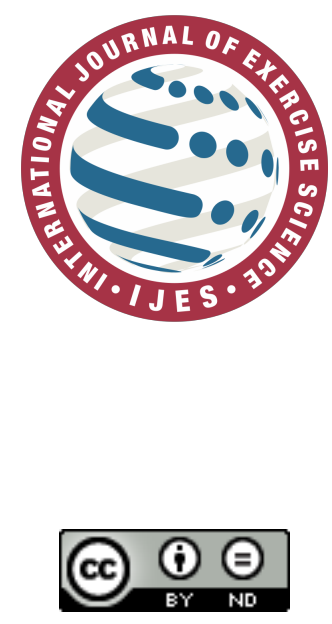